\title{
Recommandations pour une agriculture plus écologique respectant les besoins socio-écono- miques locaux, région du Menabe Central, côte ouest de Madagascar
}

\author{
Clémence Dirac Ramohavelo', Jean-Pierre Sorg', \\ Alexandre Buttler" and Michael Reinhard"'
}

\author{
Correspondence: \\ Clémence Dirac Ramohavelo \\ ETH-Zentrum, Groupe de foresterie pour le développement \\ Universitätstr 22, 8092 Zürich, Switzerland. \\ E-mail: clemence_dirac@hotmail.com
}

\begin{abstract}
RÉSUMÉ
Dans le Menabe Central (côte ouest de Madagascar), les paysages forestiers deviennent toujours plus ouverts, le taux de déforestation avoisinant les 0,7\%. La déforestation étant notamment due à des défrichements pour l'agriculture qui est la principale activité de la région, une gestion agricole écologiquement durable apparaît comme une nécessité urgente. Afin de ne pas défavoriser les populations locales vivant dans des conditions socio-économiques difficiles (81,3\% de la population malgache vivait encore en-dessous du seuil de pauvreté en 2010), cet aménagement agricole doit également viser à proposer des recommandations socio-économiquement rentables. Cette étude vise donc à confirmer I'hypothèse selon laquelle une agriculture plus écologique - permettant aux agriculteurs de se sédentariser sur leurs terres et diminuant les défrichements forestiers - qui fournirait des produits économiquement rentables sur le long terme répondrait aux nécessités socio-économiques et écologiques locales. La présente étude conclut en mettant en évidence quatre recommandations : i) La riziculture devrait être privilégiée ; ii) les techniques agro-forestières et les utilisations de fertilisants naturels devraient être encouragées ; iii) des alternatives permettant de diversifier le revenu des populations rurales, telle que la rizipisciculture, devraient être soutenues; et iv) un aménagement des surfaces sylvicoles défrichées devrait être mis en place de manière participative.
\end{abstract}

\section{ABSTRACT}

In the Central Menabe region on the west cost of Madagascar, traditional uses of forest resources create an increasingly open landscape. The current annual rate of loss of Malagasy dense dry forest, the natural forest type of the region, is $0.7 \%$. Agriculture represents the principal activity of people in Central Menabe, and the main reason for the decrease in forest cover. In the current difficult socio-economical context (81.3\% of the Malagasy population lived under the poverty line in 2010), where a threat to dry forests clearly exists, it is therefore urgent to propose scientifically-sound and participatory recommendations for ecologically sustainable and socio-economically profitable agricultural use of the Central Menabe landscape. The objective of this study is to confirm the hypothesis that a more sustainable form of agriculture - promoting farmers' permanent use of cultivated fields - would generate high value economic products, and contribute to the socio-economic and environmental needs of the region. To meet this objective, the study answers three research questions: i) What are the principal products of the villager economy? ii) what is the role of traditional agriculture in deforestation?; and, iii) what is the potential for, and the expectations of the local populations towards, more sedentary agricultural techniques? The research uses methods from both social (scoring and questionnaires) and natural sciences (inventories and measures of clearings), and was carried out in six villages representative of the Central Menabe region. Two villages mainly practiced rice cultivation, two mainly carried out slash and burn cultivation (of maize, cassava and peanut), and two practiced both slash and burn and rice cultivation. Half of the villages were situated near a national road and have thus an easy access to regional markets; the three other villages were more remote. In total 120 inhabitants were involved in 72 different scoring exercises and 288 participated in a questionnaire survey. Mann-Whitney and chi-square tests were used to test for statistical significance in observed differences.

Analyses confirm that rice is the main pillar of the villager economy in the region, and that - at the village scale - this product serves a strong commercial demand which is not always satisfied. Farmers take a weak interest in the consumption of products from slash and burn cultivation (low demand), although the majority of people that cultivate maize, cassava or peanut perceive slash and burn cultivation as a source of alimentary or financial security (subsistence or sale). Given 


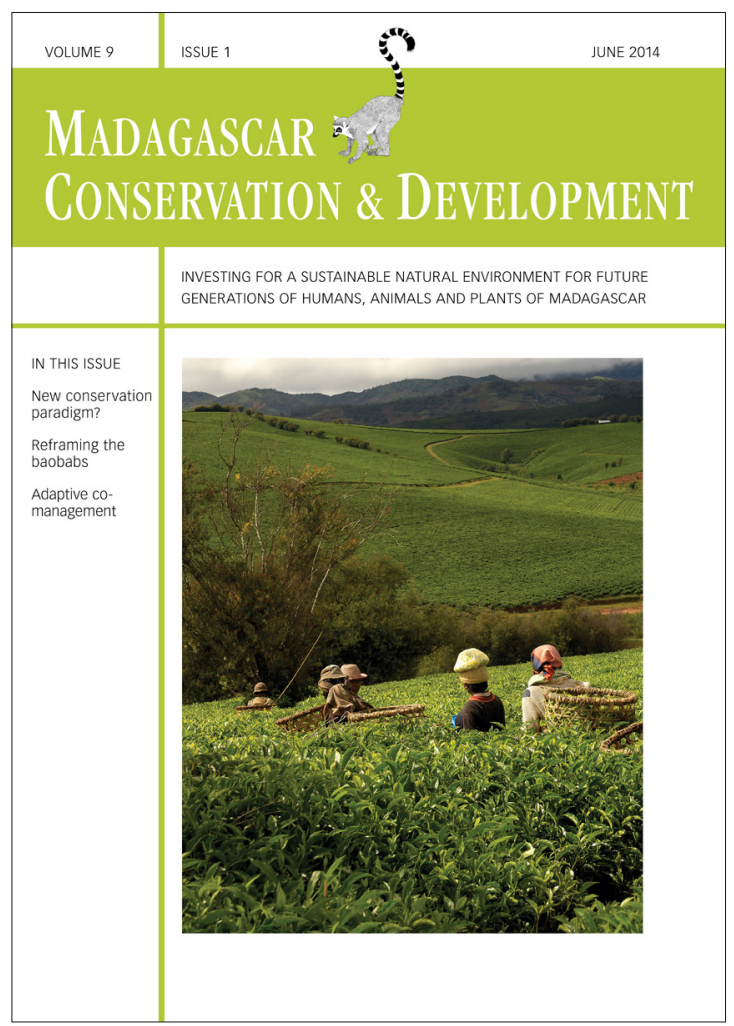

Madagascar Conservation \& Development is the journal of Indian Ocean e-Ink. It is produced under the responsibility of this institution. The views expressed in contributions to MCD are solely those of the authors and not those of the journal editors or the publisher.

All the Issues and articles are freely available at http://www.journalmcd.com

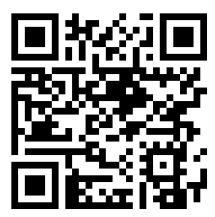

Contact Journal MCD

info@journalmcd.net for general inquiries regarding MCD funding@journalmcd.net to support the journal

Madagascar Conservation \& Development Institute and Museum of Anthropology University of Zurich

Winterthurerstrasse 190

$\mathrm{CH}-8057$ Zurich

Switzerland

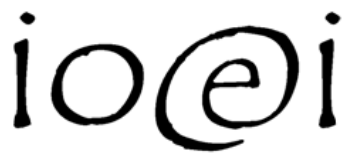

Indian Ocean e-Ink

Promoting African Publishing and Education www.ioeink.com

Missouri Botanical Garden (MBG)

Madagascar Research and Conservation Program BP 3391

Antananarivo, 101, Madagascar 
that the deforestation practice has been illegal since 1988, and the strong pressure of international biodiversity conservation organisations, the role of agriculture in deforestation is clearly a sensitive issue, almost a taboo. This study, however, confirms that nearly all slash and burn farmers (97\%) clear forest to cultivate, which exceeds the rate of clearing for rice cultivation. As the local population is open to more sedentary agricultural alternatives, four recommendations are proposed: i) Rice cultivation should be favoured, valorizing irrigation fields which are still not cultivated; ii) ethnicities who do not cultivate rice can be encouraged to cultivate trees and to use hedges and natural fertilizers. These techniques could favour farmers' settlement on cultivated fields, increasing agricultural yields and providing highly-appreciated commercial products, such as fruits; iii) new alternatives that could diversify farmers' income, such as fish farming in existing rice fields, should be favoured in order to improve livelihoods; and iv) the management of cleared forest areas should be set up in a participative way in order to legally satisfy local people's needs and the protection of natural forests.

\section{INTRODUCTION}

Dans le but de réduire la déforestation, I'attitude actuelle des programmes de conservation et des politiques forestières n'est plus d'interdire l'entrée des populations riveraines dans la forêt, parce que de telles mesures ont souvent eu et peuvent encore avoir des effets négatifs pour les communautés (Kaimowitz et Sheil 2007). Afin de respecter les besoins des populations locales, les tendances actuelles visent à proposer un aménagement multifonctionnel des espaces forestiers qui associe la conservation de la biodiversité et l'amélioration des conditions de vie des populations locales. En suivant cet élan international, la République de Madagascar - pays dans lequel $81,3 \%$ de la population vivait encore en-dessous du seuil de pauvreté en 2010, soit avec moins de US\$1,25 par jour et par personne (World Development Indicators 2014) - se lance depuis 1995 dans un processus de dévolution des droits aux communautés locales en ce qui concerne la gestion des ressources renouvelables (Rakotovao et al. 1997, Pollini et al. 2014). La situation socio-économique de Madagascar est clairement difficile. La majorité $(67 \%)$ des habitants vit en zone rurale (World Development Indicators 2014), et la faible productivité agricole, la pauvreté et le haut taux de natalité font que ce pays est classé à la $151^{\mathrm{e}}$ place (sur 187) selon l'indice de développement humain. En matière de conservation de la nature, Madagascar est internationalement considéré comme un hotspot de biodiversité (Ganzhorn et al. 2001) avec une déforestation qui menace les paysages de l'île. Dans le but de protéger les forêts des défrichements par les feux qui, liés à une forte croissance et migration des populations, sont les principales menaces qui pèsent sur les forêts (Scales 2011), le ministère des Eaux et Forêts a suspendu les délivrances de permis de défrichement en décembre 1988 (Genini 1996). Désormais, toute personne effectuant un défrichement est en infraction vis-à-vis de la loi. Dans le Menabe Central sur la côte ouest de Madagascar, la population indigène est d'ethnie Sakalava et principalement cultivatrice de riz. En raison de flux migratoires, la population indigène ne représentait guère qu'un tiers de la population totale du Menabe dans les années 1990 (Fauroux 1997). Les immigrants sont des Betsileo, des Antesaka ou des Antandroy, et ils cultivent du riz et d'autres cultures sur brûlis. II semblerait que, dans la région du Menabe Central, les premiers défrichements pour la culture sur brûlis n'auraient commencé que dans les années 1960 et seraient imputables à des ethnies migrantes (Ranaivonasy et al. 2005 voir aussi Waeber et al. 2014 et références citées). Actuellement, les populations rurales du Menabe Central dépendent fortement des terres (Genini 1996), des produits et des services forestiers (Favre 1996). Les terrains forestiers sont utilisés par les propriétaires de zébus comme sites de pâture et - une fois défrichés - ils sont convertis en terres relativement fertiles pour les cultivateurs. Les forêts de cette région sont principalement des forêts denses sèches et elles abritent de précieux produits, tels que des tubercules, des produits halieutiques, du gibier et du miel qui permettent aux populations locales de faire face à la période de soudure (Favre 1996), ainsi que du bois de construction ou encore des plantes médicinales. Elles hébergent aussi des lieux de cultes dont l'importance ne doit pas être sous-estimée. Certaines de ces différentes utilisations traditionnelles du paysage forestier du Menabe Central peuvent cependant exercer une pression considérable sur les forêts de la région. Le défrichement à des fins agricoles contribue notamment à une déforestation qui a été estimée à des valeurs variables mais vraisemblablement comprise entre 0,5\% et près de $2 \%$ par année (Scales 2011, 2012, McConnell et Kull 2014). Dans cette région, I'agriculture représente la principale activité de subsistance des populations rurales. Les produits agricoles locaux sont notamment le riz (irrigué ou pluvial), ainsi que le maïs, I'arachide et le manioc qui sont cultivés sur brûlis (Région du Menabe et le CRD Menabe 2006 In litt.). Plus de la moitié de ces produits sont consommés directement par les ménages producteurs (Dirac Ramohavelo 2009). Le manioc, le riz et le maïs font d'ailleurs partie des denrées alimentaires de base des populations rurales (Région du Menabe et le CRD Menabe 2006 In litt.).

Dans ce contexte socio-économique difficile avec une pression importante sur l'intégrité des forêts, il est capital de répondre objectivement aux attentes des acteurs locaux, en procédant de manière participative pour viser une utilisation agricole des paysages du Menabe Central qui soit socioéconomiquement rentable et écologiquement durable. Des recommandations scientifiques qui permettraient de contribuer à la réduction de la pauvreté tout en diminuant les pressions sur les paysages forestiers sont clairement attendues. Cette étude vise donc à confirmer I'hypothèse selon laquelle une agriculture plus écologique, permettant aux agriculteurs de se sédentariser sur leurs terres en diminuant dès lors les défrichements forestiers, qui fournirait des produits d'une valeur économique certaine sur le long terme répondrait tant aux nécessités socioéconomiques locales qu'aux attentes écologiques. Dans ce cadre, la présente étude a proposé de répondre à trois questions de recherche: (i) Quels sont les produits piliers de l'économie villageoise du Menabe Central ? (ii) Quelle est l'implication de I'agriculture traditionnelle dans la déforestation ? et (iii) quelles sont les potentialités et les attentes des populations locales vis-à-vis de certaines techniques agricoles plus sédentaires ?

\section{MÉTHODES}

Les villages étudiés sont ceux d'Ampataka, d'Ankoraobato, de Mandroatsy, de Marofandilia, de Kirindy et de Beroboka dans le Menabe Central (Figure 1). Les villages ont été retenus afin de constituer conjointement un échantillon représentatif du 
paysage villageois de la région. Les habitants de Beroboka et de Mandroatsy sont principalement des riziculteurs sur des terrains irrigués, ceux d'Ankoraobato et de Marofandilia pratiquent la culture sur brûlis, dans une moindre mesure la riziculture irriguée, et rarement la riziculture pluviale et seulement en ce qui concerne les habitants d'Ankoraobato. Dans les villages de Kirindy et d'Ampataka, les habitants ne pratiquent que la culture sur brûlis. Les villages retenus diffèrent aussi par leur accès aux principaux marchés de la région qui sont situés à Morondava, Belo sur Tsiribihina, Mahabo, Ankilivalo, Bemanonga et Analaiva (Figure 1). Les villages de Marofandilia, de Beroboka et de Kirindy ont un accès facile aux marchés régionaux car ils sont situés à proximité de la route nationale. Les trois autres villages sont plus difficiles d'accès, bien que le village d'Ankoraobato soit peu éloigné du marché d'Ankilivalo. La vente des produits sur les marchés régionaux requière la possession d'une charrette ou un transport par taxi brousse, de sorte que les systèmes de production visent essentiellement une agriculture de subsistance ou d'échanges de proximité. Dans la zone d'étude, le paysage varie entre des forêts denses et parfois dégradées, des zones de culture, des pâturages plus ou moins boisés et des marécages.

EXERCICES DE NOTATIONS. Les exercices de notations

(Sheil et Liswanti 2006) ont permis d'aborder les trois questions de recherche mentionnées en introduction. Tout d'abord, la question des produits piliers de l'économie villageoise a été analysée en comparant la dynamique marchande des différents produits (produits des récoltes, produits pastoraux et produits forestiers non ligneux). Les questions portaient sur les transactions commerciales locales (quels sont les produits que vous achetez/vendez/échangez sur les marchés locaux, dans les épiceries villageoises ou entre habitants ?) et sur l'offre locale des produits (quels sont les produits que vous ne trouvez pas en quantité suffisante sur les marchés locaux, dans les épiceries ou dans le village ?). Ensuite, I'implication de l'agriculture dans la déforestation a été analysée grâce à deux exercices de notations. L'un portait sur le type de surfaces cultivées (terrains cultivés l'année précédente, anciennes terres cultivées en jachère, formations dégradées, formations naturelles) et I'autre sur la durée d'utilisation des terres de culture. Un dernier exercice de notations portant directement sur les attentes

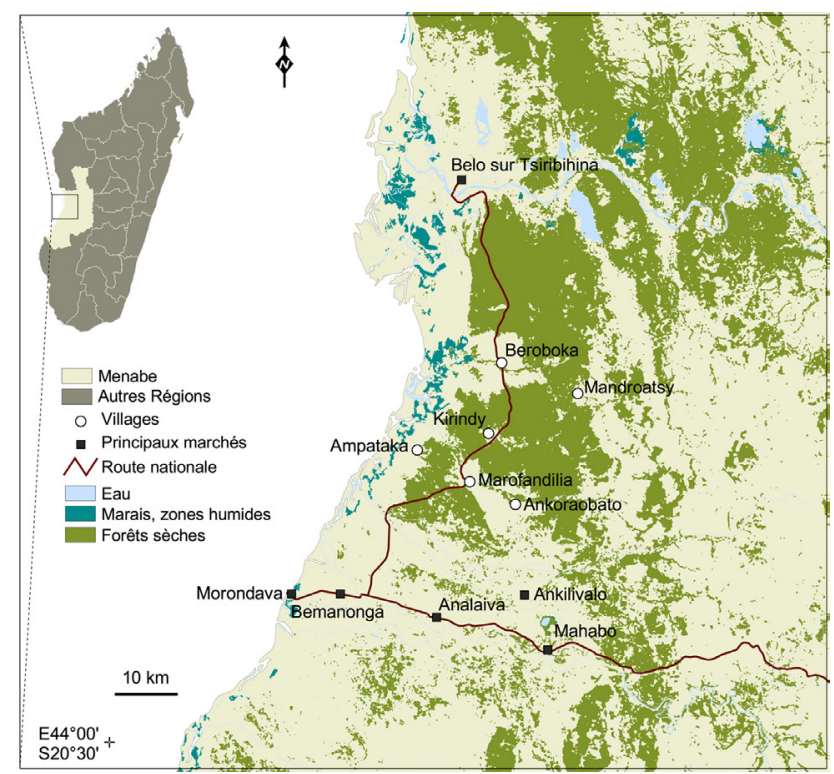

FIGURE 1. Site d'étude dans la région du Menabe. villageoises face à des techniques agricoles plus sédentaires a permis de répondre à la troisième question de recherche. Une méthode d'entretiens semi-directifs a tout d'abord été suivie afin de déterminer quelles techniques pouvaient intéresser les agriculteurs. Comme les villageois n'ont pas été réceptifs à cette méthode, en affirmant ne pas connaître d'autres techniques agricoles que celles qu'ils pratiquaient, dix techniques ont été suggérées aux villageois, et ces derniers ont quantifié leur intérêt relatif pour chacune d'elles.

Dans les six villages, CDR et un traducteur ont organisé ces exercices. Cette équipe a profité de son expérience, CDR ayant vécu dans les différents villages pendant plus de trois mois, et le traducteur étant originaire de la région, pour choisir des groupes de participants représentatifs, en fonction de l'ethnie, de l'âge et de la classe sociale. Sachant que la plupart des femmes des villages malgaches n'ont pas l'habitude d'exprimer leurs idées devant des hommes, les exercices ont été réalisés dans chaque village une fois avec un groupe d'hommes et une fois avec un groupe de femmes. Un groupe se composait d'une dizaine de personnes; au total, 20 personnes par village ont été questionnées, ce qui représentait entre $1,6 \%$ et $5 \%$ de la population des villages. Cent jetons ont été distribués à chaque participant. Une question était posée à tout le groupe. Chaque participant répondait à la question en posant les jetons dans une ou plusieurs case(s) réponse(s), selon l'importance accordée à la case réponse. Une case-réponse " autres » était à disposition.

Les résultats ont été testés statistiquement au moyen du test $\mathrm{U}$ de Mann-Whitney. Ce test a été retenu car d'une part, les données récoltées avec les exercices de notations n'étaient pas nombreuses en ne totalisant guère que 12 groupes différents, et d'autre part, les données n'étaient pas appariées, la rubrique " autres » n'ayant pas été considérée dans les analyses statistiques.

QUESTIONNAIRES. Les enquêtes au moyen des

questionnaires (cf. Supplementary Material) concernant I'implication de l'agriculture dans la déforestation ont été réalisées auprès de 288 ménages vivant dans les six villages (48 questionnaires par village, ce qui représente en moyenne plus du $40 \%$ des ménages des villages). Étant donné que les maisons des ménages d'une même ethnie se regroupent dans un village, le choix des ménages s'est fait en fonction de l'emplacement géographique des maisons, afin d'obtenir un échantillon de ménages ethniquement représentatif du village. L'analyse des questionnaires s'est focalisée sur la surface annuelle défrichée par ménage, ainsi que sur le pourcentage d'agriculteurs qui pratiquent la culture sur brûlis, le pourcentage de cultivateurs sur brûlis et de riziculteurs qui défrichent, et le pourcentage de défrichements réalisés dans différentes formations dont les forêts naturelles ou anthropisées possédant des espèces ligneuses forestières non pionnières, les formations d'espèces ligneuses ou arécacées pionnières comme ziziphus mauritiana ou Fernandoa madagascariensis, et les formations buissonnantes ou herbeuses. Pour appréhender dans quelle mesure la culture sur brûlis diffère de la riziculture dans la déforestation, le test du chi carré a été utilisé. Les cultivateurs sur brûlis qui cultivent également du riz n'ont pas été considérés dans cette analyse.

DÉNOMBREMENT DES DÉFRICHEMENTS. Afin de

trianguler les résultats portant sur les défrichements obtenus par les questionnaires, les défrichements à des fins 
agricoles autour de chaque village étudié ont été dénombrés, après identification des différentes techniques de défrichement. L'analyse s'est alors portée sur une estimation de la surface défrichée, mesurée directement sur le terrain, et sur le pourcentage de défrichements dans chaque type de formations.

Afin de ne pas gêner les acteurs locaux, les dénombrements ont été réalisés dans la plus grande discrétion en respectant les activités locales. Lors des rencontres avec des défricheurs, une discussion informelle et agréable portant sur le défrichement précédait tout travail d'enquête. La plupart du temps, les agriculteurs participaient volontiers à la discussion et décrivaient comment ils procédaient sans jamais se sentir menacés.

Le dénombrement des surfaces défrichées a été réalisé sur un rayon d'environ trois kilomètres autour du village, pour des raisons pratiques et logistiques, sachant que ces défrichements pourraient ne pas correspondre à ceux plus profondément enfouis dans la forêt (Blanc-Pamard et al. 2005). Au-delà de trois kilomètres, des photos aériennes précises auraient été nécessaires. Les défrichements destinés à 'nettoyer' des terres agricoles qui étaient utilisées au cours de l'année ou l'année précédente n'ont pas été comptabilisés. Chaque parcelle dénombrée a été mesurée et décrite en considérant le type de formations défrichées, les espèces ligneuses dominantes ou intéressantes - un guide local identifiait les espèces ligneuses - les mesures des diamètres des plus gros arbres, et le but supposé du défrichement.

\section{RÉSULTATS ET DISCUSSION}

LES PRODUITS PILIERS DE L'ÉCONOMIE VILLAGEOISE DU

MENABE CENTRAL. L'impact commercial de l'agriculture

dans le milieu rural est illustré dans les figures 2 et 3. Quarante pourcent des transactions commerciales que les villageois effectuent concernent quatre produits agricoles principaux, à savoir le riz, le maïs, le manioc et l'arachide, qui représentent donc globalement les produits piliers de l'économie locale. Dans les détails, les achats et les ventes de riz représentent environ 20\% du commerce rural (Figure 2), et sont statistiquement plus importants que ceux des autres produits présents sur les marchés ruraux $(U: p<0,01)$. Les achats et les ventes des produits issus de la culture sur brûlis représentent globalement environ 20\% du commerce rural (Figure 2), les ventes et achats de manioc (8\%), de maïs (6\%) et d'arachides (6\%), et ne sont statistiquement pas plus importants que ceux des autres produits considérés $(U: p>0,05)$. La différence majeure entre le riz et les produits de la culture sur brûlis concerne les achats $(U: p<0,01)$ et non les ventes $(U: p>0,05)$. En effet, les achats de riz sont nettement supérieurs à ceux des produits issus de la culture sur brûlis (Figure 2). Avec le lait, les légumes, les produits halieutiques et les fruits, le riz fait partie des principaux produits manquants sur les marchés ruraux (Figure $3, U: p<0,01$ ). Selon la population locale, I'offre des produits issus de la culture sur brûlis est cependant suffisante (Figure $3, \cup: p<0,01$ ).

Les résultats montrent que le riz joue un rôle central dans l'économie villageoise et présente de grands atouts économiques avec de nombreux achats et une offre qui est considérée comme insuffisante. Le développement commercial de ce produit pourrait donc avoir un effet positif sur la diminution de la pauvreté. Au niveau régional, la riziculture représente déjà I'un des axes stratégiques de développement économique et de réduction de la pauvreté (Région du Menabe et CRD Menabe

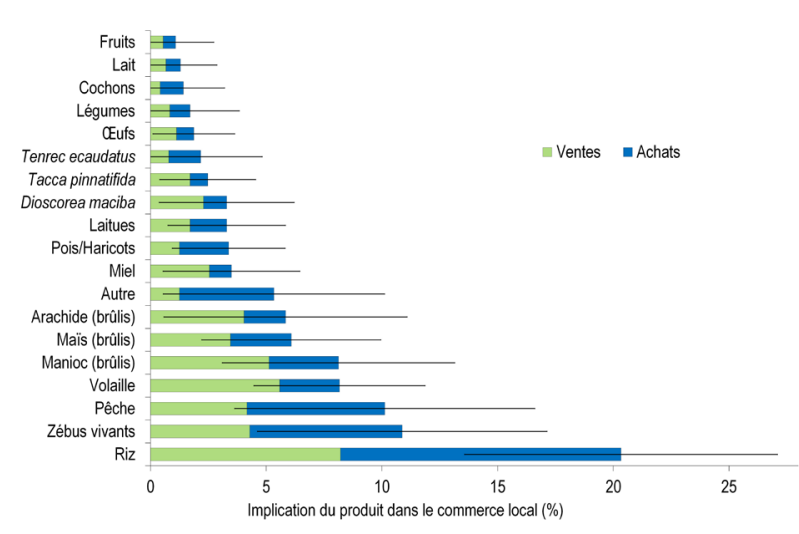

FIGURE 2. Importance relative des transactions de produits du commerce rural. ( $n=12$ groupes de 10 personnes ; afin que la somme des pourcentages attribués aux transactions de tous les produits considérés soit égale à $100 \%$ et que la transaction d'un même produit acheté par une personne et vendu par une autre ne soit pas comptée double, les pourcentages octroyés aux ventes et aux achats ont tous été divisés par un facteur 2)

2006 In litt.). Rappelons cependant que de 1944 à 1994, les rivières du Menabe Central se sont taries, rendant I'utilisation de certaines terres rizicoles difficile (Tache 1994 cité dans Raharimalala et al. 2010). Le manque d'eau évoqué par certains agriculteurs (obs. pers.) laisse penser que la situation ne s'est pas améliorée depuis les années 1990. Malgré cela, les terres irrigables de Mandroatsy ne sont pas toutes valorisées aujourd'hui (obs. pers.). Ceci pourrait s'expliquer par le caractère héritable des rizières, et donc leur accès difficile pour les migrants ou certains jeunes agriculteurs; traditionnellement, les champs de culture s'acquièrent en effet soit par défrichement de parcelles forestières soit par héritage. Certaines rizières sont cependant parfois louées à des étrangers (obs. pers.), ce qui permet de valoriser des terrains qui, sinon, ne seraient pas cultivés. Une autre explication du cas de Mandroatsy peut être trouvée dans le fait que le village est reculé en étant ainsi guère attractif pour les migrants et les jeunes générations.

Les résultats montrent que l'offre des produits issus de la culture sur brûlis est jugée suffisante. Sachant que 81,3\% de la population malgache vivait en-dessous du seuil de pauvreté en 2010 (World Development Indicators 2014), ce résultat pourrait refléter le fait que la population locale ne manifeste qu'un faible intérêt pour la consommation de ces produits. On sait en effet que d'une manière générale, la population de Madagascar préfère consommer du riz plutôt que des produits tels que le manioc ou le maïs, mais que tous les habitants n'ont

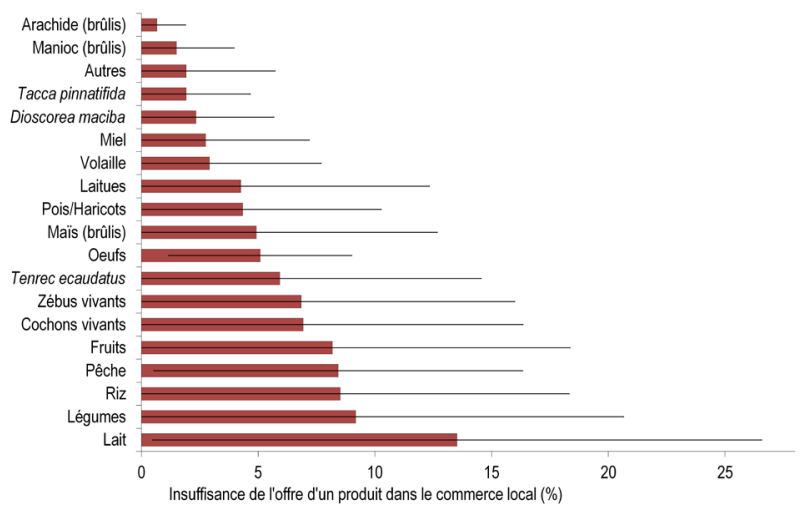

FIGURE 3. Insuffisance relative de I'offre des produits des marchés ruraux. ( $n=12$ groupes de 10 personnes; la rubrique " autre " comprend des produits tels que le pain, les produits de beauté, les meubles) 
pas la possibilité de cultiver du riz (terrains irrigables rares ou non valorisés, question des droits d'accès, manque de savoirfaire pour certains groupes). Cette préférence se retrouve clairement dans nos résultats avec une quantité de riz acheté qui est statistiquement plus grande que celle de maïs ou de manioc. Toutefois, les produits issus de la culture sur brûlis sont commercialement importants, car leurs ventes représentent pour les ménages ruraux un moyen d'acheter du riz.

Les exercices de notations montrent que la quantité de produits halieutiques présente sur les marchés locaux est insuffisante (Figure 3, $U: p<0,01$ ), alors que la pisciculture semble revêtir un intérêt local considérable (obs. pers.). Élever des poissons dans les rizières irriguées actuelles (Frei et Becker 2005) pourrait remédier au manque de produits halieutiques dans le milieu rural. Encore marginale, la rizipisciculture existe déjà sur les Hautes-Terres malgaches, avec des résultats encourageants (Kos et al. 1993).

L'IMPLICATION DE L'AGRICULTURE DANS LA

DÉFORESTATION. Une grande majorité des cultivateurs sur brûlis (environ 97\%) affirment défricher des formations d'âges divers pour cultiver, ce qui est significativement plus important $\left(X^{2}: p<0,01\right)$ que le pourcentage $(55 \%)$ de riziculteurs défrichant les mêmes formations pour cultiver du riz irrigué ou pluvial. Dans le Menabe Central, cette différence s'explique du fait que les cultivateurs de maïs, d'arachide et de manioc pratiquent une culture itinérante, alors que la riziculture pluviale ou irriguée est une culture sédentaire, de sorte qu'il est moins fréquent d'observer des défrichements destinés à la conversion en terres rizicoles.

Le développement de la filière du riz pourrait donc non seulement avoir un effet positif sur la diminution de la pauvreté, mais il pourrait également présenter un avantage pour la biodiversité. En effet, après quelques années d'utilisation, les agriculteurs sur brûlis sont souvent forcés de laisser leurs terres en jachère, car la fertilité du sol diminue drastiquement. Plus particulièrement en ce qui concerne le maïs pour lequel la production chute de $80 \%$ après quelques années de culture sur le même sol (Réau 2002, Blanc-Pamard et al. 2005). Utilisée de manière extensive (à petite échelle sur de grandes surfaces boisées), la culture itinérante peut permettre une recomposition d'espaces arborés sur des terres en friches. Mais dans le Menabe Central, ce type de culture itinérante se solde par la déforestation en raison du grand nombre de personnes qui la pratique (taux de natalité élevé et forte immigration). Certaines terres défrichées sont également maintenues en zones ouvertes par le pâturage (Dirac Ramohavelo 2009).

Les résultats des exercices de notations montrent que $53 \%$ des terres cultivées sont des parcelles qui avaient déjà été cultivées l'année précédente, que 22,5\% étaient issues de défrichements d'anciennes terres en jachère, que 19\% correspondaient à diverses formations dégradées et que 5,5\% des terres agricoles étaient des formations naturelles. Selon les questionnaires, plus du $69 \%$ des agriculteurs pratiquent la culture de maïs, d'arachide et de manioc sur brûlis; les autres sont principalement riziculteurs. D'après les agriculteurs, la surface annuelle moyenne des défrichements est de 1,60 \pm 1,53 ha par ménage défricheur. Globalement, $61 \%$ des formations défrichées sont des formations dégradées d'espèces ligneuses ou arécacées pionnières de Ziziphus mauritiana ou Fernandoa madagascariensis, 27\% des formations herbeuses ou buissonnantes et $12 \%$ des forêts naturelles ou dégradées formées d'espèces ligneuses non pionnières telles que Commiphora sp. ou Poupartia sylvatica. Suivant le dénombrement des défrichements, deux stratégies de défrichement ont été observées avec d'une part les cas où l'agriculteur étend son champ de culture en défrichant la forêt limitrophe, et d'autre part les cas où l'agriculteur défriche en une fois une surface qui lui servira globalement de champs de culture. La surface moyenne des défrichements agricoles recensés est de 0,89 $\pm 1,11$ ha. D'après le dénombrement effectué, 69\% des défrichements agricoles concernent des forêts intactes ou dégradées abritant des espèces ligneuses non pionnières telles que Commiphora sp ou Poupartia sylvatica, 18\% des formations d'espèces ligneuses ou arécacées pionnières tels que Ziziphus mauritiana, Fernandoa madagascariensis, Bismarkia nobilis ou Grewia sp., et 11\% des formations herbeuses ou buissonnantes. Le dénombrement des défrichements et les questionnaires aboutissent à des valeurs différentes en ce qui concerne la surface des défrichements. Les questionnaires ont pu fournir des résultats biaisés car dans le cas de grands défrichements, les ménages qui en ont les moyens payent d'autres personnes du village pour participer au travail (communication personnelle). Certaines surfaces ainsi défrichées ont donc pu être comptabilisées plusieurs fois dans les questionnaires et surestimer la surface totale défrichée annuellement. De plus, il est possible que pour répondre au questionnaire, certains agriculteurs aient considéré le nettoyage des champs de culture comme un acte de défrichement. Dans cet article, le dénombrement des défrichements semble donc fournir le résultat le plus fiable.

L'estimation de la fréquence des défrichements des différentes formations végétales s'est avérée difficile. En effet, les exercices de notations et les questionnaires aboutissent à des résultats concordants entre eux mais contradictoires comparés à ceux issus du dénombrement des défrichements. Chacune des méthodes présente cependant des inconvénients. Dans la mesure où le thème des défrichements est un sujet délicat qui devient presque tabou dans le Menabe Central, suite à la pression des organisations actives dans la conservation de la biodiversité et à l'interdiction étatique de défricher les zones boisées depuis 1988, les populations locales dissimulent sans doute certaines de leurs activités, ce qui a un effet direct sur les informations recueillies par les questionnaires et les exercices de notations. Le fait que la règlementation des défrichements soit la proposition qui remporte le moins de succès chez les agriculteurs (Figure 4) confirme le malaise à ce sujet. En ce qui concerne le dénombrement, le rayon de trois kilomètres est certainement insuffisant pour disposer d'une image complète

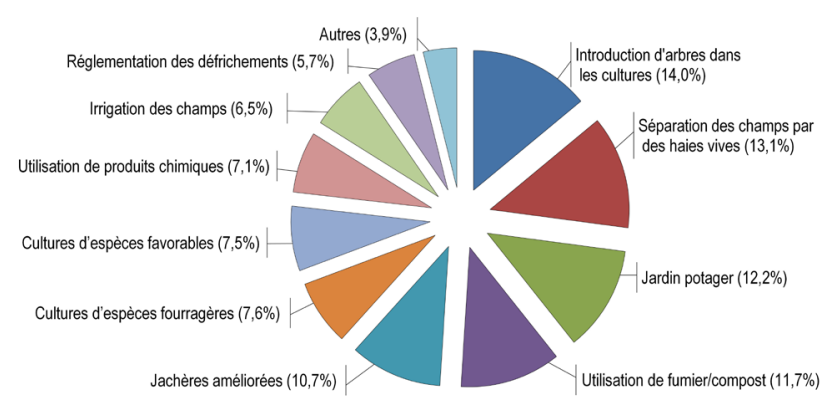

FIGURE 4. Présentation des préférences locales vis-à-vis de techniques agricoles visant à freiner la déforestation. ( $n=12$ groupes de 10 personnes) 
des formations brûlées, d'autant que même à l'intérieur de ce périmètre l'exhaustivité ne peut être atteinte car certains brûlis peuvent être cachés dans la forêt naturelle ou dans les formations dégradées. Ces résultats contradictoires montrent la nécessité de recourir à d'autres méthodes de prise de données afin de connaître la fréquence des défrichements effectués dans chaque type de formation. Par exemple, un suivi des activités des ménages ou l'utilisation de photos aériennes précises semblent représenter des alternatives mieux appropriées pour estimer le pourcentage de défrichement selon les formations végétales. Cependant, ces méthodes nécessitent un suivi à long terme.

LES POTENTIALITÉS ET ATTENTES LOCALES CONCERNANT UNE AGRICULTURE PLUS SÉDENTAIRE. Les exercices de notations (Figure 4) montrent que les agriculteurs sont intéressés par l'arboriculture fruitière, la séparation des champs par des haies vives et l'enrichissement des sols agricoles avec des fertilisants naturels. Ces trois techniques pourraient favoriser la sédentarisation des agriculteurs sur les terres de cultures en améliorant les rendements agricoles, et en diminuant ainsi les défrichements, donc l'avancée de la déforestation. En outre, I'arboriculture fournirait des produits commerciaux appréciés, tels les fruits qui sont - avec le lait, le riz, les produits halieutiques et les légumes - insuffisamment présents sur les marchés ruraux (Figure $3, U: p<0,01$ ). Des études participatives visant les potentialités locales des techniques agroforestières sont toutefois nécessaires, car les recherches actuelles sur ce sujet ont rarement impliqué les populations rurales. Elles se sont focalisées sur des essais en champs avec les villageois mais sans préalablement considérer les attentes ou les besoins locaux. L'enrichissement des sols agricoles avec des fertilisants naturels nécessite également une évaluation participative de ses potentialités, car en raison du mode d'élevage extensif ou semi-extensif (Région du Menabe et CRD Menabe 2006 In litt.), il n'y a guère de fumier à récolter.

Toujours selon les exercices de notations (Figure 4), la culture maraîchère semble également représenter un intérêt majeur pour les agriculteurs, et l'analyse sur les transactions commerciales montre que les légumes font partie des produits les moins commercialisés (Figure $2, U: p<0.01$ ). En outre, I'offre de légumes est considérée comme insuffisante (Figure 3, U: p $<0.01)$. Le commerce de légumes semble donc représenter une alternative prometteuse dans le Menabe Central, bien que certains essais locaux de culture maraîchère n'aient pas été couronnés de succès (com. pers.).

\section{CONCLUSION}

Conformément aux résultats de la présente recherche, quatre recommandations d'aménagement agricole peuvent être proposées aux acteurs de l'aide au développement, aux politiciens locaux et aux organisations internationales de conservation de la biodiversité. II en ressort premièrement que le commerce du riz présente un avantage économique et écologique dont il faudrait tirer parti dans une plus grande mesure. La riziculture devrait être privilégiée en aménageant - par exemple - les terres irrigables qui ne sont pas cultivées actuellement. Cependant, le caractère héritable des rizières - qui rend ces terres difficilement accessibles aux jeunes et aux migrants - doit être considéré et respecté ; certaines terres pourraient par exemple être Iouées, étant donné que cette pratique semble être acceptée dans la région. De plus, il est important de considérer également le fait que certaines ethnies ne sont traditionnellement pas prédisposées à cultiver du riz.

Les résultats de cette étude présentent la rizipisciculture comme une alternative qui pourrait diversifier le revenu des populations rurales et qui devrait donc être encouragée. Illukpitiva et Yanagida (2008) ont en effet montré l'impact de la diversification des revenus ruraux sur I'amélioration des conditions de vie des populations tout comme sur la protection des ressources forestières. Cependant, les populations locales ne possèdent souvent pas les ressources matérielles et les connaissances spécifiques nécessaires à certaines nouvelles activités potentiellement prometteuses (Pollini et al. 2014) ; elles doivent donc être soutenues dans ces démarches.

Troisièmement, cette étude met en évidence que les populations locales sont ouvertes à certaines alternatives agricoles leur permettant de se sédentariser davantage. Soutenues par les agriculteurs eux-mêmes, les techniques agroforestières et I'utilisation de fertilisants naturels pourraient y contribuer en améliorant les rendements agricoles et en fournissant des produits commerciaux appréciés, tels que les fruits. La sédentarisation ne répondrait cependant pas au problème lié à la pression démographique et donc au besoin de nouvelles terres pour les immigrants et les jeunes sans héritage. Étant donné que traditionnellement, une personne ne peut devenir propriétaire d'une terre que par héritage ou par défrichement, il est nécessaire que l'État malgache reconnaisse légalement le droit au défrichement (voir ci-dessous) par exemple pour les anciens défrichements qui ont 20 ans ou plus et qui ont retrouvé un niveau de fertilité acceptable (Raharimalala et al. 2010).

Pour terminer, la présente recherche a confirmé le malêtre des populations rurales quant à la situation actuelle liée aux défrichements. Elle a également mis en lumière le fait que la fréquence des défrichements demeurait élevée. Dans un tel contexte, la pertinence d'un aménagement raisonné et participatif des défrichements dans les différentes formations est évidente. Bien que les différents acteurs du paysage forestier du Menabe Central n'aient pas tous les mêmes attentes et que les défrichements agricoles relèvent quasiment du tabou, la discussion devrait être privilégiée pour converger vers une règlementation participative des défrichements visant à satisfaire à la fois les besoins des populations et la protection des forêts naturelles.

\section{REMERCIEMENTS}

Les auteurs remercient le North-South Center de Zurich qui par son programme RFPP a soutenu financièrement le projet dans lequel cet article s'insère. Nous remercions tous nos partenaires locaux, et en particulier I'ESSA Eaux et Forêts, Lanto Andriambelo et le CNFEREF, ainsi que les assistants de recherche qui ont participé à la récolte de données et bien entendu les villageois pour leur participation active à nos nombreux entretiens et exercices de notations. Pour terminer, nous aimerions remercier les trois rapporteurs anonymes qui ont permis d'améliorer une version initiale de ce manuscrit et Ralisa Andriamahavita pour les figures.

\section{RÉFÉRENCES}

Blanc-Pamard, C., Milleville, P., Grouzis, M., Lasry, F. et Razanaka, S. 2005. Une alliance de disciplines sur une question environnementale : La déforestation en forêt des Mikea (sud-ouest de Madagascar). Natures Sciences Sociétés 13, 1: 7-20. (doi:10.1051/nss:2005002) 
Dirac Ramohavelo, C. 2009. Stratégies villageoises dans la gestion des paysages forestiers, Menabe Central Madagascar. Thèse de doctorat non publiée, École Polytechnique Fédérale de Lausanne, Suisse. Disponible <http://infoscience.epfl.ch/record/135636/files/EPFL_TH4400.pdf>

Fauroux, E. 1997. Les représentations du monde végétal chez les Sakalava du Menabe. In: Milieux et Sociétés dans le Sud-ouest de Madagascar J.-M. Lebigre, E. Fauroux, B. Moizo, J. Taillade, P. Vasseur, C. HenryChartier et P. Henry (eds.), pp. 7-26. Collection îles et Archipels, Bordeaux, France.

Favre, J.-C. 1996. Traditional utilization of the forest. In: Ecology and Economy of a Tropical Dry Forest in Madagascar, Primate Report 46-1. J. U. Ganzhorn \& J.-P. Sorg (eds.), pp 33-40. Erich Goltze GmbH \& Co KG, Göttingen, Allemagne.

Frei, M. \& Becker, K. 2005. Integrated rice-fish culture: Coupled production saves resources. Natural Resources Forum 29, 2: 135-143. (doi:10.1111/j.1477-8947.2005.00122.x)

Ganzhorn, J. U., Lowry, P. P., Schatz, G. E. \& Sommer, S. 2001. The biodiversity of Madagascar: one of the world's hottest hotspots on its way out. Oryx 35, 4: 346-348. (doi:10.1111/j.1365-3008.2001.00201.pp.x)

Genini, M. 1996. Deforestation. In: Ecology and Economy of a Tropical Dry Forest in Madagascar, Primate Report 46-1. J. U. Ganzhorn \& J.-P. Sorg (eds), pp 49-55. Erich Goltze GmbH \& Co KG, Göttingen, Allemagne.

Illukpitiya, P. \& Yanagida, J. 2008. Role of income diversification in protecting natural forests: evidence from rural households in forest margins of Sri Lanka. Agroforestry Systems 74, 1: 51-62. (doi:10.1007/s10457-0089153-2)

Kaimowitz, D. \& Sheil, D. 2007. Conserving what and for whom? Why conservation should help meet basic human needs in the tropics. Biotropica 39, 5: 567-574. (doi:10.1111/j.1744-7429.2007.00332.x)

Kos, C., Ramaroson, H. et Janssen, J. 1993. Étude de I'impact quantitatif des activités rizipiscicoles et piscicoles dans les régions pilotes $d u$ Vakinankaratra et du Betsileo, Campagne 1991-1992. <http://www.fao. org/docrep/field/003/AB852F/AB852F00.htm\#TOC> téléchargé le 10 décembre 2008.

Le Bourdiec, F. 1980. Le développement de la riziculture dans I'ouest malgache. In: Changements Sociaux dans I'Ouest Malgache. R. Waast, E. Fauroux, B. Schlemmer, F. Le Bourdiec, J.-P. Raison et G. Dandoy (eds.), pp. 133-152. Collection Mémoires, 90, ORSTOM, Paris.

McConnell, W. J. \& Kull, C. A. 2014. Deforestation in Madagascar; Debates over the island's forest cover and challenges of measuring forest change. In: Conservation and Environmental Management in Madagascar. I. Scales (ed.), pp 65-104. Routledge, Taylor \& Francis Group, London and New York.

Moat, J. \& Smith, P. 2007. Atlas of the vegetation of Madagascar. The Royal Botanic Gardens Kew, Richmond, U.K.

Pollini, J., Hockley, N., Muttenzer, F. D. \& Ramamonjisoa, B. S. 2014. The transfer of natural resource management rights to local communities. In: Conservation and Environmental Management in Madagascar. I. Scales (ed.), pp 172-192. Routledge, Taylor \& Francis Group, London and New York.

Raharimalala, O., Buttler, A., Dirac Ramohavelo, C., Razanaka, S., Sorg, J.-P. \& Gobat, J.-M. 2010. Soil-vegetation patterns in secondary slash and burn successions in Central Menabe, Madagascar. Agriculture, Ecosystems \& Environment 139, 1-2: 150-158. (doi:10.1016/j. agee.2010.07.013)

Rakotovao, A. S., Razafindrabe M. et Bertrand A.1997. Vers la gestion communautaire locale des feux de végétation à Madagascar: L'élaboration de Dina types pour la gestion locale des feux dans diverses régions de Madagascar. Akon'ny Ala 20: 8-22.

Ranaivonasy, J., Durbin, J. et Raharinjanahary, H. 2005. Étude de I'Évolution des Différents Régimes de Gestion des Aires Protégées à Madagascar (A Review of Development of Different Management Regimes for the New Protected Areas in Madagascar). Étude de Cas : la Future Aire Protégée du Menabe Central. Disponible <http://agro.univ-antananarivo.mg/gouvernance_ap/file/menabe.pdf>

Réau, B. 2002. Burning for zebu: the complexity of deforestation issues in western Madagascar. Norwegian Journal of Geography 56, 3: 219-229. (doi:10.1080/00291950260293048)

Scales, I. R. 2011. Farming at the forest frontier: Land use and landscape change in Western Madagascar, 1896-2005. Environment and History 17: 499-524. (doi:10.3197/096734011X13150366551481)
Scales, I. R. 2012. Lost in translation: conflicting views of deforestation, land use and identity in western Madagascar. The Geographical Journal 178 1: 67-79. (doi:10.1111/j.1475-4959.2011.00432.x)

Sheil, D. \& Liswanti, N. 2006. Scoring the importance of tropical forest landscapes with local people: Patterns and insights. Environmental Management 38, 1: 126-136. (doi:10.1007/s00267-005-0092-7)

Waeber, P. O., Wilmé, L., Ramamonjisoa, B., Garcia, C., Rakotomalala, D. Rabemananjara, Z. H., Kull, C., Ganzhorn, J. U. \& Sorg, J.-P. 2014 (In press). Dry Forests in Madagascar, neglected and under pressure. International Forestry Review.

World Development Indicators 2014. Disponible <http://data.worldbank.org/ indicator/SI.POV.DDAY>

\section{SUPPLEMENTARY MATERIAL. \\ AVAILABLE ONLINE ONLY.}

Table S1. Questionnaire. 